\title{
Mapas conceptuales vs. Resúmenes: diferencias en las modalidades de construcción colaborativa
}

\section{Conceptuals Maps vs Summaries: Differences in the Modalities of Collaborative Construction}

\author{
Nestor Daniel Roselli* \\ Pontificia Universidad Católica Argentina, Argentina \\ ORCID: https://orcid.org/0000-0002-7313-4566 \\ Juliana Cardoni \\ Pontificia Universidad Católica Argentina, Argentina. \\ ORCID: https://orcid.org/0000-0003-2142-207X
}

Received 02-24-20 Revised 05-25-20 Accepted 08-08-20 On line 10-25-20

*Correspondence

Email: nestorroselli@uca.edu.ar
Cite as:

Roselli, N., \& Cardoni, J. (2020). Mapas conceptuales vs. Resúmenes: diferencias en las modalidades de construcción colaborativa. Propósitos y Representaciones, 8(3), e429. doi: http://dx.doi.org/10.20511/pyr2020.v8n3.429 


\section{Resumen}

Se comparan dos recursos didácticos: el mapa conceptual y el resumen, entendidos como sistemas externos de representación cognitiva. En el enfoque teórico utilizado, tales sistemas externos no son entendidos como mera externalización del pensamiento, sino, inversamente, como tipos de actividad representacional que formatean a la propia cognición. En este sentido, los mapas y los resúmenes tendrían efectos cognitivos distintos. Pero esta investigación va más allá: tales efectos diferenciales serían también psicosociales, formateando la interacción sociocognitiva cuando la actividad se realiza colaborativamente. El diseño experimental compara cuatro condiciones de dos submuestras idénticas, en las que se balancea tanto la tarea que 18 díadas de alumnos universitarios deben realizar (mapa y resumen), como los dos textos-fuente empleados. El análisis compara las producciones individuales con la colectiva de cada díada, centrándose en las modalidades de la construcción colectiva a partir de las producciones individuales. Así, se distinguen tres modalidades básicas: homogénea, heterogénea simétrica y heterogénea asimétrica. El análisis diferencia entre aspectos cognitivos o de contenido, y aspectos específicamente formales. De manera descriptiva, los datos muestran una tendencia en el sentido de la hipótesis planteada, mostrando que ambos sistemas externos moldean de manera distinta la interacción sociocognitiva.

Palabras Clave: Mapas Conceptuales; Resúmenes; Modalidades de Colaboración; Interacción sociocognitiva; Sistemas externos de representación.

\section{Summary}

Two teaching resources are compared: the conceptual map and the summary, understood as external systems of cognitive representation. In the theoretical approach used, such external systems are not understood as mere outsourcing of thought, but, conversely, as types of representational activity that format the cognition itself. In this sense, maps and summaries would have different cognitive effects. But this research goes further: such differential effects would also be psychosocial, formating sociocognitive interaction when the activity is carried out in a collaborative way. The experimental design compares four conditions from two identical subsamples, in which both the task that 18 dyads of university students must perform (map and summary) and the two source-texts used are balanced. The analysis compares the individual productions with the collective of each dyad, focusing on the modalities of collective construction based on the individual productions. Thus, three basic modalities are distinguished: homogeneous, symmetrical heterogeneous and asymmetrical heterogeneous. The analysis differentiates between cognitive or content aspects, and specifically formal aspects. Descriptively, data shows a tendency in the sense of the hypothesis raised, showing that both external systems shape differently the sociocognitive interaction.

Keywords: Conceptuals Maps; Summaries; Modalities of sociocognitive collaboration;

Sociocognitive interaction; External representation systems.

\section{Introducción}

En la práctica educativa es común el uso de múltiples recursos didácticos: los apuntes de clase, los resúmenes, los mapas conceptuales, los gráficos, las tablas estadísticas, los esquemas, los dibujos, entre otros. Estos recursos pueden ser proporcionados por el docente o un texto-fuente, o ser realizados por los propios alumnos, ya sea a solicitud del docente o espontáneamente. En todos los casos el objetivo es facilitar la apropiación de información y/o la comprensión conceptual, o sea el aprendizaje de conocimientos. Pese a este uso extendido, llama la atención el desconocimiento que hay en los medios educativos de los aspectos cognitivos involucrados en tales recursos. Éste es un aspecto que la psicología cognitiva de la educación pretende dilucidar, aportando elementos teóricos sobre las implicancias cognitivas de cada uno de ellos. Precisamente, en los últimos tiempos ha surgido un prolífico campo de investigación sobre los llamados sistemas externos de representación simbólica, con una impronta centrada tanto en una 
psicología del desarrollo, como en una psicología de la educación (Martí, 2003; Martí y Pozo 2000; Puy Pérez-Echeverría, Martí y Pozo, 2010). Básicamente, el énfasis en tales sistemas implica una revalorización de la "exterioridad" cognitiva, superando la idea de que éstos cumplen una mera función instrumental.

El uso del término "sistemas externos" se entiende por su contraposición con el de procesos cognitivos internos o pensamiento propiamente dicho. Ahora bien, "externo" alude tanto a una condición de materialidad objetiva, como al carácter social de dichas producciones, en el sentido que tienen y permiten visibilidad intersubjetiva. Sin duda, el lenguaje oral es el sistema externo de representación más importante creado por la cultura. Vygotski le otorga un papel central en tanto instrumento psicológico (Kozulin, 2000), en su clásica distinción entre herramientas (dirigidas a modificar la naturaleza) y sistemas de signos lingüísticos (fundamento de la acción comunicativa). Es bien sabida la función estructurante del pensamiento que este representante fundacional del enfoque histórico-cultural otorgó al habla. En este sentido, es común la contraposición entre el enfoque teórico del psicólogo ruso, que sostenía que lo intersubjetivo (el habla) precede a lo intrasubjetivo (el pensamiento) y el de Piaget, más centrado en la construcción cognitiva individual que realiza el propio sujeto a partir de sus acciones sobre el medio (Martí, 2000; Rivière, 1985; Wertsch, 1988). Si bien es cierto que la intersubjetividad para Vygotski reposa básicamente en el sistema de signos externos llamado lenguaje, es justo reconocer que la postura del psicólogo ruso, que con razón se la suele definir como socioconstructivista, está lejos del determinismo lingüístico mecanicista postulado por Sapir, Whorf y otros representantes de esa corriente psicolingüística (Wertsch, 1991), para quienes el pensamiento es esencialmente reproducción interiorizada del lenguaje.

Más allá de esta polémica, lo cierto es que la externalidad del lenguaje oral no es la misma que la del lenguaje escrito. La primera es esencialmente temporal; la segunda es espacial, lo cual le otorga un carácter de objetividad innegable (Martí, 2003). Pero la escritura no es el único sistema externo de representación simbólica. Existen otros sistemas notacionales, tales como el matemático (aritmético y geométrico), el musical, el químico y el gestual-icónico, los que configuran distintos tipos de "escritura". Finalmente, existen representaciones simbólicas externas de segundo orden, que combinan elementos de los sistemas representacionales (de primer orden) nombrados, como por ejemplo las tablas y gráficos estadísticos, las matrices de datos, las partituras musicales para canto, las figuras o esquemas, las historietas o comics, los calendarios, las maquetas, las fotos y dibujos (figurativos o no), los mapas geográficos y, por supuesto, los mapas conceptuales y los resúmenes escritos de ideas centrales. Más allá de esta vasta variedad de sistemas representacionales, lo importante es analizar los aspectos cognitivos involucrados en cada uno, en especial el formateo cognitivo a que cada uno da lugar. Esto implica considerar que tales sistemas no tienen una simple función representacional de un concepto o una idea, sino que no son cognitivamente neutros; bien por el contrario, implican un particular formateo del pensamiento como tal (Martí, 2017; Pozo, 2017; Puy Pérez-Echeverría et al., 2010). En otras palabras, estos sistemas externos no son meros disfraces representacionales; formatean el conocimiento mismo como actividad mental o interna. Así, se podría hablar de un proceso de interiorización cognitiva, paralelo al de exteriorización simbólica. En este sentido, hacer un mapa conceptual de una episteme ofrecida no conduciría al mismo tipo de apropiación epistémica que un resumen. En otras palabras, no se trata de testear una simple variabilidad representacional de un mismo contenido epistémico (Roselli, 1999). Se trata más bien de lo contrario, es decir, de un formateo lógico-sintáctico diferencial del conocimiento que se intenta aprender (interiorizar), producto de recurrir a distintos sistemas externos de representación del conocimiento ofrecido. La idea de base es que los distintos sistemas externos de representación cognitiva implican estructuras sintácticas diferentes, las que convocan a diferentes sintaxis de pensamiento; así, el formato cognitivo exterior sería la antesala del formato cognitivo interior.

Como se dijo, el papel cognitivamente estructurante de los sistemas externos de representación ha dado lugar a numerosas investigaciones (Brizuela y Cayton, 2010; Castellaro y Roselli, 2018; Lorenzo y Pozo, 2010; Puy Pérez-Echeverría, Postigo y Marín, 2010; Rodríguez, 
Martí y Salsa, 2018; Salsa y Gariboldi, 2017). Sin embargo, no es este determinismo cognitivo intraindividual el objetivo central del presente estudio. En cambio, lo que ha sido poco abordado son los efectos diferenciales que tales sistemas representacionales tienen en la interacción sociocognitiva entre pares cuando se trata de una producción cognitiva colaborativa. De lo que se trata es del formateo sociocognitivo que los distintos sistemas representacionales producen en un uso colectivo. En este caso se trata de confrontar dos sistemas representacionales, el mapa conceptual y el resumen, en cuanto a las modalidades de interacción colaborativa asociadas a ambas. En este sentido, la presente investigación es complementaria de una anterior (Roselli, 2017), cuyo objetivo fue analizar las distintas modalidades de interacción sociocognitiva en la realización diádica de mapas conceptuales a partir de una episteme proporcionada por el docente. Dicha investigación permitió diferenciar tres modalidades básicas de la relación entre las producciones individuales de una díada y la producción conjunta: a) Homogénea (coincidencia entre las producciones individuales de los dos sujetos que se traslada a la producción colectiva, ya sea incorporando u omitiendo elementos); b) Heterogénea Simétrica (a partir de producciones individuales bien diferentes, construcción de la producción colectiva sumando o restando elementos de ambas en forma equitativa); c) Heterogénea Asimétrica (partiendo de producciones individuales diferentes, construcción de un mapa colectivo en base al aporte preponderante de uno de los sujetos en detrimento de la incorporación de elementos del mapa individual del otro). En esta ocasión, el fundamento de la elección, con fines comparativos, de los dos sistemas de representación cognitiva mencionados radica en que ambos difieren en cuanto a los aspectos formales básicos: uno es preponderantemente gráfico, en tanto el otro es exclusivamente lingüístico. En el campo educativo, el mapa conceptual, más que el resumen, ha merecido apreciable atención (Aguilar Tamayo, 2006; Notorio, 1992), quizás por utilizar el grafema como soporte de la palabra. De todas maneras, esta atención ha sido puesta en tanto recurso didáctico dirigido a facilitar el aprendizaje, no como formato representacional externo determinante de una particular estructura sintáctica del pensamiento, y menos con intenciones comparativas con un formato representacional exclusivamente lingüístico.

El objetivo de la presente investigación es comparar la relación entre producciones individuales y colectivas referidas a la realización de mapas conceptuales (MC) y resúmenes (RES). Se intentan describir las modalidades de producción sociocognitiva en ambos casos, hipotetizando que, aun tratándose de las mismas díadas (muestras idénticas), hay diferencias significativas en lo que hace a las modalidades de articulación cognitiva intersubjetiva. Ello en base al presupuesto teórico antes aludido de que los sistemas externos de representación (en este caso de segundo orden) formatean no sólo el conocimiento que se aprende, sino la propia interacción sociocognitiva. Es en el análisis de este formateo de la interacción colaborativa donde se pone el acento, centrándose en la estructuración específicamente cognitiva, más que en variables psicosociales de corte socioafectivo o de dinámica interaccional.

Como el análisis se focaliza en la relación entre producciones individuales y colectiva, no contándose con el registro del proceso de construcción colaborativa, no se entra a discriminar entre negociación y consenso intersubjetivo auténtico, como bien lo hacen Kirschner (2007) y Mejía-Arauz, Rogoff, Dayton y Henne-Ochoa (2018). El análisis es sólo de los mapas y resúmenes realizados individual y colectivamente, siendo el objetivo constatar de qué manera las sintaxis diferenciales de ambos sistemas representacionales determinan distintas "sintaxis" sociorelacionales. Estas "sintaxis" interaccionales se refieren a las distintas modalidades sociocognitivas mencionadas: homogeneidad, heterogeneidad simétrica y heterogeneidad asimétrica.

\section{Método}

\section{Muestra}

La muestra estuvo constituida por 9 díadas, repartidas en dos submuestras (4 y 5 díadas cada una), todo/as alumno/as universitarias de la Carrera de Psicopedagogía de una universidad privada de Buenos Aires. Se trató de un muestreo no-probabilístico ya que las submuestras fue el resultado 
de una división de los alumnos de un curso de una asignatura en dos grupos, actividad que estuvo a cargo del profesor de la misma. La conformación de las díadas al interior de cada submuestra se realizó espontáneamente, según la afinidad socioafectiva existente entre los alumnos. El curso era poco numeroso (18 alumnos), lo que explica el carácter reducido de las dos submuestras. Queda claro que este tamaño muestral no da mucho margen para la aplicación de pruebas de estadística inferencial, por lo que cabe otorgarle al estudio un objetivo básicamente descriptivoexploratorio.

\section{Diseño}

Como es común en la investigación en contextos educativos, el diseño fue cuasi-experimental, tanto por las características referidas del muestreo, como por la equivalencia relativa de los dos textos-fuente utilizados, ya que, si bien eran del mismo autor y temática parecida, la extensión del texto B (28 unidades cognitivas) era algo mayor que la del texto A (17 unidades cognitivas). Las unidades cognitivas son los elementos cognitivos más simples o moleculares que es posible reconocer en un texto epistémico, manteniendo una unidad de sentido o significación. Dichas unidades se reconocen operacionalmente por constituir una oración, separada de la precedente y siguiente por un punto o un punto-coma. El total de las unidades cognitivas de un texto escrito resulta de la descomposición del mismo en las distintas oraciones (separadas por puntos) y suboraciones (separadas por punto-comas).

\section{Primera Fase}

CONDICIÓN 1 (Submuestra 1): Aprendizaje individual de texto expositivo A ("si quieren pueden marcar, hacer notas o subrayar en el texto, pero no tomar apuntes fuera del texto") (15') / MC individual con el texto-fuente (15') / MC en díada sin el texto-fuente, pero con el MC individual $\left(15^{\prime}\right)$.

CONDICIÓN 2 (Submuestra 2): Aprendizaje individual de texto expositivo A ("si quieren pueden marcar, hacer notas o subrayar en el texto, pero no tomar apuntes fuera del texto") (15') / Resumen individual con el texto-fuente (15') / Resumen en díada sin el texto-fuente, pero con el resumen individual (15').

Segunda Fase (consecutiva a la anterior)

CONDICIÓN 3 (Submuestra 2): Aprendizaje individual de texto expositivo B ("si quieren pueden marcar, hacer notas o subrayar en el texto, pero no tomar apuntes fuera del texto") (15') / MC individual con el texto-fuente (15') / MC en díada sin el texto-fuente, pero con el MC individual $\left(15^{\prime}\right)$.

CONDICIÓN 4 (Submuestra 1): Aprendizaje individual de texto expositivo B ("si quieren pueden marcar, hacer notas o subrayar en el texto, pero no tomar apuntes fuera del texto") (15' / Resumen individual con el texto-fuente (15') / Resumen en díada sin el texto-fuente, pero con el resumen individual (15').

Nota. Cada condición tuvo tres subfases. Cada subfase duró 15 minutos. Los sujetos de la condición 1 y 4 fueron los mismos; los sujetos de la condición 2 y 3 fueron los mismos. Cabe advertir que, dado que la cantidad de unidades cognitivas de los textos-fuente A y B era diferente (17 y 28 respectivamente), la comparación entre MC y RES, en los casos que involucraran distintos textos-fuente, demandaba la relativización de los valores, dividiendo éstos por 17 o 28 según correspondiera.

\section{Procedimiento}

En una primera fase el docente-investigador implementó las condiciones 1 y 2 , en cada submuestra, en base al texto-fuente expositivo A, solicitando Mapas Conceptuales en el primer caso y Resúmenes en el segundo. 
En una segunda fase el docente-investigador implementó las condiciones 3 y 4, en cada submuestra, en base al texto-fuente expositivo B, solicitando Mapas Conceptuales en el primer caso y Resúmenes en el segundo.

Previamente a la segunda subfase de cada condición, el docente-investigador explicaba, según el caso, lo que es un mapa conceptual y un resumen.

\section{Resultados}

Las tablas 1, 2, 3 y 4 presentan los resultados correspondientes a las condiciones del diseño. Exhiben la cantidad de unidades cognitivas presentes en las producciones individuales (discriminadas por sujeto) y colectivas, mostrando el aporte de los sujetos individuales a la producción colectiva. Estos valores están categorizados según que haya habido coincidencias de contenido entre los aportes de cada sujeto de la díada al producto colectivo (Sumatoria homogénea), que no haya habido coincidencias, o sea que dichos aportes han sido diferentes (Sumatoria heterogénea), que haya habido pérdida de unidades cognitivas individuales coincidentes (Resta homogénea), que haya habido pérdidas diferenciales de unidades cognitivas no-coincidentes (Resta heterogénea), y, por último, que en la producción colectiva haya habido unidades cognitivas inéditas o exclusivas de la misma, no presentes en las producciones individuales (Construcción).

Tabla 1

Contribución de los sujetos de las díadas de la submuestra 1 a la producción colectiva de MC (Condición 1)

\begin{tabular}{ccccccccc}
\hline & $\begin{array}{c}\text { Sumatoria } \\
\text { Homogéne } \\
\text { a (SHo) }\end{array}$ & $\begin{array}{c}\text { Sumatoria } \\
\text { Heterogénea } \\
\text { (SHe) }\end{array}$ & $\begin{array}{c}\text { Resta } \\
\text { Homogénea } \\
\text { (RHo) }\end{array}$ & $\begin{array}{c}\text { Resta Heterogénea } \\
\text { (RHe) }\end{array}$ & $\begin{array}{c}\text { Construcc } \\
\text { ión } \\
\text { (C) }\end{array}$ & $\begin{array}{c}\text { Total } \\
\text { General }\end{array}$ \\
\hline & & $\begin{array}{c}\text { Sujeto } \\
\text { A }\end{array}$ & $\begin{array}{c}\text { Sujeto } \\
\text { B }\end{array}$ & & $\begin{array}{c}\text { Sujeto } \\
\text { A }\end{array}$ & $\begin{array}{c}\text { Sujeto } \\
\text { B }\end{array}$ & & \\
\hline Díada 1 & 3 & 2 & 0 & 2 & 0 & 2 & 1 & 10 \\
Díada 2 & 10 & 0 & 1 & 0 & 2 & 1 & 0 & 14 \\
Díada 3 & 3 & 6 & 0 & 0 & 0 & 2 & 0 & 11 \\
Díada 4 & 4 & 0 & 2 & 0 & 1 & 0 & 0 & 7 \\
\hline TOTAL & $\mathbf{2 0}$ & $\mathbf{8}$ & $\mathbf{3}$ & $\mathbf{2}$ & $\mathbf{3}$ & $\mathbf{5}$ & $\mathbf{1}$ & $\mathbf{4 2}$ \\
\hline $\begin{array}{c}\text { PORCENTAJE } \\
\text { (\%) }\end{array}$ & $\mathbf{4 7 , 6 2}$ & $\mathbf{1 9 , 0 5}$ & $\mathbf{7 , 1 4}$ & $\mathbf{4 , 7 6}$ & $\mathbf{7 , 1 4}$ & $\mathbf{1 1 , 9 0}$ & $\mathbf{2 , 3 8}$ & $\mathbf{1 0 0}$ \\
\hline
\end{tabular}

Nota. "Sumatoria homogénea": unidades cognitivas que estaban presentes en ambas producciones individuales (coincidentes) y que aparecen en la producción colectiva; "Sumatoria heterogénea": unidades cognitivas no-coincidentes de las producciones individuales y que aparecen en la producción colectiva; "Resta homogénea": unidades cognitivas que estaban presentes en ambas producciones individuales (coincidentes) pero que no aparecen en la producción colectiva; "Resta heterogénea": unidades cognitivas no-coincidentes de las producciones individuales y que no aparecen en la producción colectiva; "Construcción": unidades cognitivas que aparecen en la producción colectiva y no estaban en ninguna de las producciones individuales. 
Tabla 2

Contribución de los sujetos de las díadas de la submuestra 1 a la producción colectiva de RES (Condición 4)

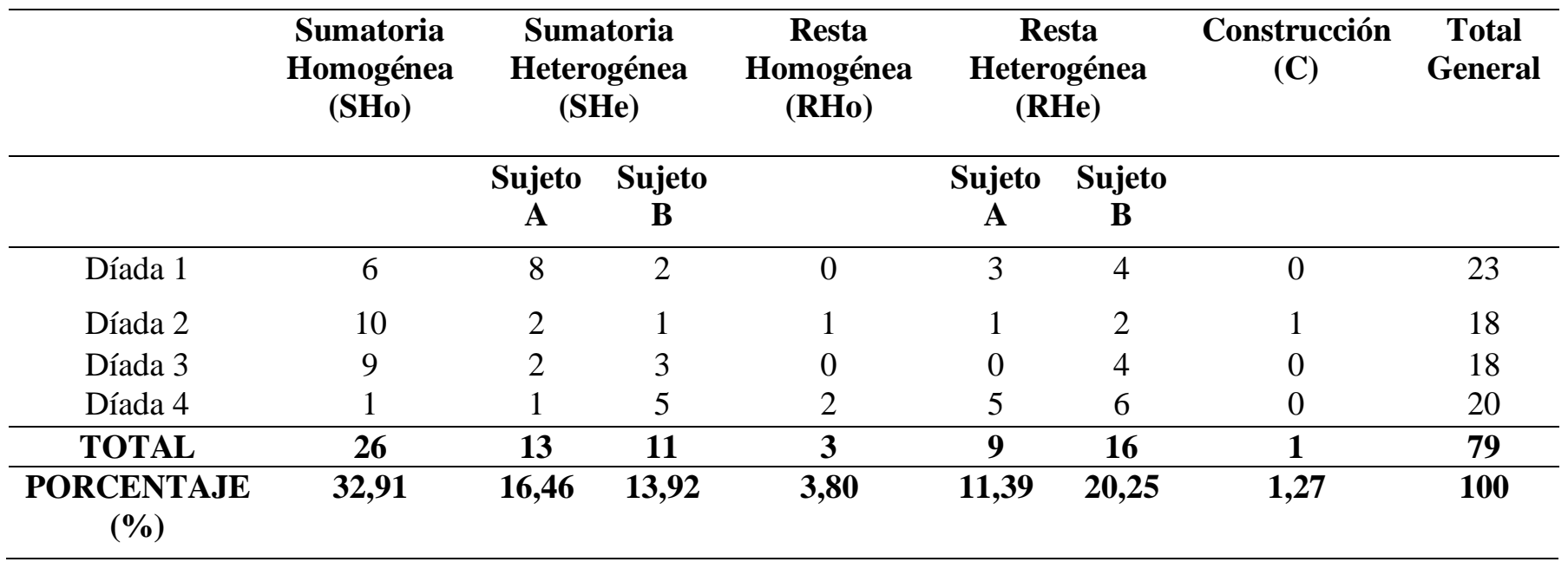

Nota. Cfr. nota de Tabla 1.

Tabla 3

Contribución de los sujetos de la submuestra 2 a la producción colectiva de MC (Condición 3)

\begin{tabular}{|c|c|c|c|c|c|c|c|c|}
\hline & $\begin{array}{c}\text { Sumatoria } \\
\text { Homogénea } \\
\text { (SHo) }\end{array}$ & $\begin{array}{r}\text { Sum } \\
\text { Heter } \\
\text { (S }\end{array}$ & $\begin{array}{l}\text { toria } \\
\text { génea } \\
\text { Ie) }\end{array}$ & $\begin{array}{c}\text { Resta } \\
\text { Homogénea } \\
\text { (RHo) }\end{array}$ & $\begin{array}{r}\mathbf{R} \\
\text { Heter } \\
(\mathbf{R}\end{array}$ & $\begin{array}{l}\text { sta } \\
\text { génea } \\
\text { He) }\end{array}$ & $\begin{array}{l}\text { Construcción } \\
\text { (C) }\end{array}$ & $\begin{array}{c}\text { Total } \\
\text { General }\end{array}$ \\
\hline & & $\begin{array}{c}\text { Sujeto } \\
\text { A }\end{array}$ & $\begin{array}{c}\text { Sujeto } \\
\text { B }\end{array}$ & & $\begin{array}{c}\text { Sujeto } \\
\text { A }\end{array}$ & $\begin{array}{c}\text { Sujeto } \\
\text { B }\end{array}$ & & \\
\hline Díada 1 & 8 & 0 & 3 & 0 & 4 & 0 & 0 & 15 \\
\hline Díada 2 & 10 & 1 & 5 & 0 & 0 & 0 & 0 & 16 \\
\hline Díada 3 & 6 & 5 & 1 & 0 & 1 & 3 & 0 & 16 \\
\hline Díada 4 & 2 & 2 & 2 & 2 & 2 & 2 & 0 & 12 \\
\hline Díada 5 & 5 & 2 & 1 & 1 & 0 & 9 & 0 & 18 \\
\hline TOTAL & 31 & 10 & 12 & 3 & 7 & 14 & $\mathbf{0}$ & 77 \\
\hline $\begin{array}{c}\text { PORCENTAJE } \\
(\%)\end{array}$ & 40,26 & 12,99 & 15,58 & 3,90 & 9,09 & 18,19 & $\mathbf{0}$ & 100 \\
\hline
\end{tabular}

Nota. Cfr. nota de Tabla 1. 
Tabla 4

Contribución de los sujetos de las díadas de la submuestra 2 a la producción colectiva de RES (Condición 2)

\begin{tabular}{|c|c|c|c|c|c|c|c|c|}
\hline & \multirow[t]{2}{*}{$\begin{array}{c}\text { Sumatoria } \\
\text { Homogéne } \\
\text { (SHo) }\end{array}$} & \multicolumn{2}{|c|}{$\begin{array}{c}\text { Sumatoria } \\
\text { Heterogénea } \\
\text { (SHe) }\end{array}$} & \multirow[t]{2}{*}{$\begin{array}{c}\text { Resta } \\
\text { Homogéne } \\
\text { (RHo) } \\
\end{array}$} & \multicolumn{2}{|c|}{$\begin{array}{c}\text { Resta } \\
\text { Heterogénea } \\
\text { (RHe) } \\
\end{array}$} & \multirow[t]{2}{*}{$\begin{array}{c}\text { Construcció } \\
\mathbf{n} \\
(\mathbf{C}) \\
\end{array}$} & \multirow[t]{2}{*}{$\begin{array}{c}\text { Total } \\
\text { General }\end{array}$} \\
\hline & & $\begin{array}{c}\text { Sujet } \\
\text { A }\end{array}$ & $\begin{array}{c}\text { Sujet } \\
\text { B }\end{array}$ & & $\begin{array}{c}\text { Sujet } \\
\text { A }\end{array}$ & $\begin{array}{c}\text { Sujet } \\
\text { B }\end{array}$ & & \\
\hline Díada 1 & 0 & 0 & 6 & 4 & 2 & 3 & 0 & 15 \\
\hline Díada 2 & 2 & 1 & 6 & 0 & 0 & 1 & 0 & 10 \\
\hline Díada 3 & 4 & 1 & 1 & 1 & 6 & 0 & 0 & 13 \\
\hline Díada 4 & 8 & 1 & 2 & 0 & 2 & 0 & 0 & 13 \\
\hline Díada 5 & 7 & 0 & 2 & 0 & 1 & 1 & 0 & 11 \\
\hline TOTAL & 21 & 3 & 17 & 5 & 11 & 5 & $\mathbf{0}$ & 62 \\
\hline $\begin{array}{c}\text { PORCENTAJE } \\
(\%)\end{array}$ & 33,87 & 4,84 & 27,42 & 8,06 & 17,74 & 8,06 & $\mathbf{0}$ & 100 \\
\hline
\end{tabular}

Nota. Cfr. nota de Tabla 1.

El análisis de los datos, a partir de los valores primarios presentados precedentemente (tablas 1, 2, 3 y 4), tiene 4 partes: a) Análisis de la cantidad de unidades cognitivas recuperadas del texto-fuente por las producciones individuales y colectiva de cada díada; b) Análisis diádico de la homogeneidad-heterogeneidad del contenido cognitivo (unidades cognitivas) aportado por cada sujeto a la producción colectiva; c) Análisis diádico de la heterogeneidad simétricaasimétrica del contenido cognitivo (unidades cognitivas) aportado por cada sujeto a la producción colectiva; d) Análisis de los aspectos formales de las producciones individuales y colectiva de cada díada. Está claro que, en todos los casos, la comparación recurrente es entre RES y MC.

La secuencia lógica del análisis es la siguiente. En primer lugar, se trata de confrontar el volumen o riqueza cognitiva de las producciones individuales con el de las respectivas producciones colectivas, esto en función de la cantidad de unidades cognitivas recuperadas del texto-fuente (A). En segundo lugar, se trata de determinar el grado de homogeneidad y heterogeneidad de las producciones colectivas a partir del contenido (coincidente o nocoincidente) aportado por cada miembro de la díada (B). En tercer lugar, el objetivo es determinar cuánto de la heterogeneidad que se constata en el aporte no-coincidente de los miembros es simétrica y cuanto asimétrica (C). Finalmente, se analizan, no ya los aspectos de contenido, sino los específicamente formales de las producciones individuales y las respectivas producciones colectivas.

\section{Análisis de la cantidad de unidades cognitivas recuperadas del texto-fuente por las producciones individuales y colectiva de cada díada}

Tabla 5

Cantidad de unidades cognitivas del respectivo texto-fuente recuperadas por las producciones individuales y colectivas de las condiciones 1 y 4 (submuestras idénticas)

\begin{tabular}{|c|c|c|c|c|c|c|}
\hline \multirow[b]{3}{*}{ Díada 1} & \multicolumn{3}{|c|}{ Mapas Conceptuales (Texto A) } & \multicolumn{3}{|c|}{ Resúmenes (Texto B) } \\
\hline & \multicolumn{2}{|c|}{ Individual } & \multirow{2}{*}{$\begin{array}{c}\text { Colectivo } \\
6\end{array}$} & \multicolumn{2}{|c|}{ Individual } & \multirow{2}{*}{$\begin{array}{c}\text { Colectivo } \\
16\end{array}$} \\
\hline & 7 & 7 & & 17 & 12 & \\
\hline Díada 2 & 12 & 12 & 11 & 14 & 14 & 14 \\
\hline Díada 3 & 9 & 5 & 9 & 11 & 16 & 14 \\
\hline Díada 4 & 5 & 6 & 6 & 9 & 14 & 7 \\
\hline Total & \multicolumn{2}{|c|}{63} & 32 & \multicolumn{2}{|c|}{107} & 51 \\
\hline $\begin{array}{c}\text { Total } \\
\text { relativizado }\end{array}$ & \multicolumn{2}{|c|}{$\mathbf{0 , 4 6}$} & 0,47 & \multicolumn{2}{|c|}{0,48} & $\mathbf{0 , 4 6}$ \\
\hline
\end{tabular}


Nota. El "Total relativizado" es el coeficiente que resulta de dividir los valores absolutos de recuperación de unidades cognitivas por el total de unidades cognitivas del texto-fuente correspondiente $(\mathrm{A}=17 ; \mathrm{B}=28)$. Como ya se dijo, esta relativización es necesaria con fines comparativos dado que la cantidad de unidades cognitivas de los dos textos-fuentes no es la misma.

\section{Tabla 6}

Cantidad de unidades cognitivas del respectivo texto-fuente recuperadas por las producciones individuales y colectivas de las condiciones 2 y 3 (submuestras idénticas)

\begin{tabular}{|c|c|c|c|c|c|c|}
\hline \multirow[b]{3}{*}{ Díada 1} & \multicolumn{3}{|c|}{ Mapas Conceptuales (Texto B) } & \multicolumn{3}{|c|}{ Resúmenes (Texto A) } \\
\hline & \multicolumn{2}{|c|}{ Individual } & \multirow{2}{*}{$\begin{array}{c}\text { Colectivo } \\
11\end{array}$} & \multicolumn{2}{|c|}{ Individual } & \multirow{2}{*}{$\frac{\text { Colectivo }}{6}$} \\
\hline & 12 & 11 & & 6 & 13 & \\
\hline Díada 2 & 11 & 15 & 16 & 3 & 9 & 9 \\
\hline Díada 3 & 12 & 10 & 12 & 12 & 6 & 6 \\
\hline Díada 4 & 8 & 8 & 6 & 11 & 10 & 11 \\
\hline Díada 5 & 8 & 16 & 8 & 8 & 10 & 9 \\
\hline Total & \multicolumn{2}{|c|}{111} & 53 & \multicolumn{2}{|c|}{88} & 41 \\
\hline $\begin{array}{c}\text { Total } \\
\text { relativizado }\end{array}$ & \multicolumn{2}{|c|}{$\mathbf{0 , 4 0}$} & $\mathbf{0 , 3 8}$ & \multicolumn{2}{|c|}{$\mathbf{0 , 5 2}$} & 0,48 \\
\hline
\end{tabular}

Nota. Cfr. nota de Tabla 5.

Los datos muestran que la recuperación de unidades cognitivas por parte de RES (individuales y colectivos) es levemente mayor que la de MC, o sea que en MC hay una tendencia a un mayor filtrado de información $(0,86$ y 0,85 de recuperación individual y colectiva respectivamente frente a 1,00 y 0,94 de RES, siempre con valores relativizados). En otras palabras, RES tiende a ser más exhaustivo, lo cual es entendible ya que RES es exclusivamente lingüístico y secuencial; en cambio MC combina palabra con grafema y es estructural. Esto se aprecia en el comportamiento de ejecución de ambos, que si bien su análisis no formó parte del objetivo de la investigación, resulta interesando mencionarlo como dato etnográfico complementario: se escribe RES a medida que se va leyendo el texto-fuente; en cambio en MC primero se lee todo el texto y luego se construye el mapa. A igual conclusión se llega si comparamos los valores absolutos (individual y colectivo) de las dos submuestras de MC en conjunto, con los de las dos submuestras de RES en conjunto: 174 para MC individual y 85 para MC colectivo frente a 195 para RES individual y 92 para RES colectivo. Con todo, el test de Wilcoxon no otorga significatividad estadística a ambas diferencias, tanto calculado sobre valores absolutos $(T=54 / \mathrm{N}=16 / \mathrm{p}>0.05$ en las producciones individuales; $\mathrm{T}=20 / \mathrm{N}=9 / \mathrm{p}>0.05$ en las producciones colectivas), como sobre valores relativizados ( $\mathrm{T}=52 / \mathrm{N}=18 / \mathrm{p}>0.05$ en las producciones individuales; $\mathrm{T}=24 / \mathrm{N}=9 / \mathrm{p}>0.05$ en las producciones colectivas); en cambio, el test de signos otorga significatividad estadística a la diferencia entre MC y RES individuales para valores absolutos $(X=5 / \mathrm{N}=16 / \mathrm{p}<0.05)$ y relativizados $(X=2 / \mathrm{N}=18 / \mathrm{p}<0.05)$, no así a la diferencia en las producciones colectivas. Es obvio que lo reducido del tamaño muestral no da mucho margen para la inferencia estadística, aunque no inhabilita detectar tendencias. De todas maneras, este formateo cognitivo diferencial no es el objetivo central de este trabajo; sí lo es los análisis que siguen.

\section{Análisis diádico de la homogeneidad-heterogeneidad del contenido cognitivo (unidades cognitivas) aportado por cada sujeto a la producción colectiva}

Las Tablas 7 y 8 muestra los valores de homogeneidad-heterogeneidad de MC y RES para cada submuestra. 
Tabla 7

Homogeneidad-heterogeneidad entre las producciones individual y colectiva de la submuestra 1 para MC y RES

\begin{tabular}{cccc}
\hline & \multicolumn{3}{c}{ MAPAS CONCEPTUALES } \\
\cline { 2 - 4 } Díada 1 & Homogéneo & Heterogéneo & Razón \\
\cline { 2 - 4 } Díada 2 & 5 & 4 & 1,25 \\
Díada 3 & 10 & 4 & 2,50 \\
Díada 4 & 3 & 8 & 0,36 \\
\hline Total & 4 & 3 & 1,33 \\
\hline Total de Razón & $\mathbf{2 2}$ & $\mathbf{5 , 4 4}$ \\
\hline & \multicolumn{19}{|c}{} \\
Díada 1 & Homogéneo & Heterogéneo & Razón \\
Díada 2 & 6 & 17 & 0,35 \\
Díada 3 & 11 & 6 & 1,83 \\
Díada 4 & 9 & 9 & 1,00 \\
\hline Total & 3 & 17 & 0,18 \\
\hline Total de Razón & $\mathbf{2 9}$ & $\mathbf{4 9}$ & $\mathbf{3 , 3 6}$ \\
\hline
\end{tabular}

Nota. La "Razón" se calcula dividiendo el valor de homogeneidad de cada díada por el respectivo valor de heterogeneidad. El "Coeficiente total de razón" resulta de la suma de los coeficientes parciales de razón de cada díada.

Tabla 8

Homogeneidad-heterogeneidad entre las producciones individual y colectiva de la submuestra 2 para MC y RES

\begin{tabular}{cccc}
\hline & \multicolumn{3}{c}{ MAPAS CONCEPTUALES } \\
\cline { 2 - 4 } Díada 1 & Homogéneo & Heterogéneo & Razón \\
\cline { 2 - 4 } Díada 2 & 8 & 7 & 1,14 \\
Díada 3 & 10 & 6 & 1,67 \\
Díada 4 & 6 & 10 & 0,60 \\
Díada 5 & 4 & 8 & 0,50 \\
\hline Total & 6 & 12 & 0,50 \\
\hline Total de Razón & $\mathbf{3 4}$ & $\mathbf{4 3}$ & $\mathbf{4 , 4 1}$ \\
\hline & & & Razón \\
& Homogéneo & Heterogéneo & 0,36 \\
Díada 1 & 4 & 11 & 0,25 \\
Díada 2 & 2 & 8 & 0,63 \\
Díada 3 & 5 & 8 & 1,60 \\
Díada 4 & 8 & 5 & 1,75 \\
Díada 5 & 7 & 4 & $\mathbf{4 , 5 9}$ \\
\hline Total & $\mathbf{2 6}$ & $\mathbf{3 6}$ & \\
\hline Total de Razón & & &
\end{tabular}

Nota. Cfr. nota de Tabla 7.

La suma de los coeficientes de razón entre homogeneidad y heterogeneidad de MC de las dos submuestras $(5,44+4,41=9,85)$ es mayor que los de RES $(3,36+4,59=7,95)$, lo que indica que en MC hay una tendencia a una mayor homogeneidad, siempre en relación con la heterogeneidad. La explicación posible de esto es que la focalización de MC en el nodo central de la estructura temática convoca a una mayor coincidencia, lo que no ocurriría en una recuperación más 
extendida (y por lo tanto más variable) en RES. De todas maneras hay que ser precavido con esta conclusión dado que los valores diferenciales de razón entre MC y RES en la submuestra 2 son menos distantes. Incluso si confrontamos los valores absolutos de la homogeneidad y heterogeneidad de las dos submuestras sumadas de $\mathrm{MC}$ (homogeneidad=56; heterogeneidad $=62$; $\mathrm{R}=0,90$ ) con los de los RES (homogeneidad $=55$; heterogeneidad $=85 ; \mathrm{R}=0,65$ ), se aprecia que la diferencia entre MC y RES no reside en la homogeneidad en sí misma (que es parecida), sino en la relación con la heterogeneidad, que es ostensiblemente mayor en RES, lo que es entendible dado la mayor cantidad de unidades cognitivas que recuperan los RES. Sin embargo, la probabilidad estadística con el test de Wilcoxon y el de signos para la diferencia de razón entre homogeneidad y heterogeneidad no alcanza niveles de significatividad $(\mathrm{T}=17 / \mathrm{N}=9 / \mathrm{p}>0.05$ en el primer caso y $\mathrm{X}=4 / \mathrm{N}=9 / \mathrm{p}>0.05$ en el segundo caso). Estos valores descriptivos de diferencias entre MC y RES también se constatan comparando valores absolutos y relativizados exclusivamente de heterogenidad; la heterogenidad es siempre más pronunciada en RES.

Pero hay otro aspecto que llama la atención y que es bien notorio. En varios casos no hay coincidencia intradíada entre MC y RES en cuanto a la homogeneidad y heterogeneidad. Así, en la submuestra 1, las díadas 1 y 4 muestran una tendencia a la homogeneidad en MC, pero en RES ocurre lo contrario, y la díada 3 tiene un predominio de heterogeneidad en MC que no se registra en RES. En la submuestra 2 ocurre otro tanto: las díadas 1 y 2 muestran mayor homogeneidad en MC respecto a RES, en tanto que las diadas 4 y 5 evidencian mayor heterogeneidad; exactamente lo contrario ocurre en esas mismas díadas en RES respecto a MC. Esto habla de un formateo diferencial de MC y RES en lo que hace a la dimensión homogeneidad-heterogeneidad.

\section{Análisis diádico de la heterogeneidad simétrica-asimétrica del contenido cognitivo (unidades cognitivas) aportado por cada sujeto a la producción colectiva}

Las Tablas 9 y 10 muestran los valores de simetría-asimetría de MC y RES correspondientes a las dos submuestras.

Tabla 9

Aportes heterogéneos (de sumatoria y resta) de los sujetos de cada díada de la submuestra 1 en MC y RES

\begin{tabular}{cccc}
\hline & \multicolumn{4}{c}{ MAPAS CONCEPTUALES } & \\
\hline & A & B & $\begin{array}{c}\text { Distancia o } \\
\text { Diferencia }\end{array}$ \\
\hline Díada 1 & 2 & -2 & 4 \\
Díada 2 & -2 & 0 & 2 \\
Díada 3 & 6 & -2 & 8 \\
Díada 4 & -1 & 2 & 3 \\
\hline Total Distancia & & B & \\
\hline & & RESÚMENES & Distancia o \\
& & -2 & 7 \\
& & -1 & 2 \\
\hline Díada 1 & 5 & -1 & 3 \\
Díada 2 & 1 & -1 & $\mathbf{1 5}$ \\
Díada 3 & 2 & & \\
Díada 4 & -4 & & \\
\hline Total Distancia & & &
\end{tabular}


Tabla 10

Aportes heterogéneos (de sumatoria y resta) de los sujetos de cada díada de la submuestra 2 en MC y RES

\begin{tabular}{cccc}
\hline & \multicolumn{4}{c}{ MAPAS CONCEPTUALES } & \\
\hline & A & B & $\begin{array}{c}\text { Distancia o } \\
\text { Diferencia }\end{array}$ \\
\hline Díada 1 & -4 & 3 & 7 \\
Díada 2 & 1 & 5 & 4 \\
Díada 3 & 4 & -2 & 6 \\
Díada 4 & 0 & 0 & 0 \\
Díada 5 & 2 & -8 & 10 \\
\hline Total Distancia & & & $\mathbf{2 7}$ \\
\hline & & RESÚMENES & Distancia o \\
\hline Díada 1 & B & & 5 \\
Díada 2 & -2 & 3 & 4 \\
Díada 3 & 1 & 5 & 6 \\
Díada 4 & -5 & 1 & 3 \\
Díada 5 & -1 & 2 & 2 \\
\hline Total Distancia & -1 & 1 & $\mathbf{2 0}$ \\
\hline
\end{tabular}

Ambas tablas permiten calcular la distancia o diferencia entre los aportes heterogéneos de los dos sujetos de cada díada, o sea los aportes individuales no-coincidentes sumados o restados a la producción colectiva. Se observa que las distancias son mayores en MC, lo que indica que hay mayor asimetría en la contribución no-coincidente de los dos sujetos. Esto se constata en las dos submuestras. Si sumamos los valores de ambas (MC: 27+17=44; RES: $20+15=35$ ), con lo que se controla el posible sesgo de la variabilidad de los textos-fuentes (A y B), el test de Wilcoxon y el de signos otorgan una significación estadística aceptable $(\mathrm{T}=5 / \mathrm{N}=9</ \mathrm{p} 0.05$ en el primer caso y $X=2 / \mathrm{N}=9 / \mathrm{p}<0.05$ en el segundo caso). Esto habla de un formateo psicosocial distinto de MC y RES.

Pero, al igual de lo evidenciado en el análisis de la homogeneidad-heterogeneidad del apartado anterior, en el análisis de la simetría-asimetría también se encuentran contradicciones intradíadas. Así, en la submuestra 1, el sujeto A de la díada 2 es el que registra una mayor asimetría a su favor, aspecto que se invierte en RES. A su vez, en la submuestra 2, hay contradicciones internas en las díadas 3, 4 y 5 entre MC y RES en lo que hace a los valores de asimetría de los sujetos A y B. Esto es una prueba más del formateo diferencial de la interacción sociocognitiva producto del uso de los dos sistemas externas considerados.

\section{Análisis de los aspectos formales de las producciones individuales y colectiva de cada díada.}

En el análisis de los aspectos formales de MC se tomó en cuenta: a) lugar de inicio en la hoja; b) estructura (flujo o radial); c) relación entre palabra y grafema (predominancia de uno u otro); d) tipo de grafema (rectángulo, círculo, elipse, nube); e) conectores (flecha, línea, corchete). En la Tabla 11 se presentan los aportes formales de cada miembro de la díada a la producción colectiva, en comparación con los aportes de contenido. Esto se aplica básicamente a MC porque en RES la escritura, en su aspecto formal, es uniforme ya que es aporte exclusivo de una persona: la que escribe, que siempre es uno de los miembros, hecho que salta a la vista en el análisis grafológico de la escritura. En cambio, en MC, aunque es posible reconocer quien tuvo mayor protagonismo en la confección física del MC colectivo, los elementos formales referidos pueden provenir de 
ambos sujetos. Incluso en varios casos la realización física del propio dibujo se hace en franca coautoría.

Tabla 11

Relación entre los aportes de contenido de los miembros de cada díada y los aportes formales, para MC y RES

MAPAS CONCEPTUALES

\begin{tabular}{ccccc}
\hline & \multicolumn{2}{c}{ ASPECTOS FORMALES } & \multicolumn{2}{c}{ CONTENIDO } \\
\hline & A & B & A & B \\
\hline Díada 1 & 4 & 5 & 5 & 3 \\
Díada 2 & 7 & 3 & 10 & 11 \\
Díada 3 & 3 & 5 & 9 & 3 \\
Díada 4 & 4 & 5 & 4 & 6 \\
Díada 5 & 3 & 8 & 8 & 11 \\
Díada 6 & 6 & 8 & 11 & 15 \\
Díada 7 & 5 & 3 & 11 & 7 \\
Díada 8 & 6 & 6 & 4 & 4 \\
Díada 9 & 4 & 5 & 7 & 6 \\
\hline
\end{tabular}

\section{RESÚMENES}

\begin{tabular}{ccccc}
\hline & \multicolumn{2}{c}{ ASPECTOS FORMALES } & \multicolumn{2}{c}{ CONTENIDO } \\
\hline & A & B & A & B \\
\hline Díada 1 & X & & 14 & 8 \\
Díada 2 & & $X$ & 12 & 11 \\
Díada 3 & $X$ & 11 & 12 \\
Díada 4 & $\mathrm{X}$ & & 2 & 6 \\
Díada 5 & $\mathrm{X}$ & & 0 & 6 \\
Díada 6 & & $\mathrm{X}$ & 3 & 8 \\
Díada 7 & & $\mathrm{X}$ & 5 & 5 \\
Díada 8 & & $\mathrm{X}$ & 9 & 10 \\
Díada 9 & & $\mathrm{X}$ & 7 & 9 \\
\hline
\end{tabular}

Nota. En RES se marca con una cruz el autor formal de la escritura, ya que en todos los casos dicha función fue unipersonal.

Se observa que, tanto en MC, como en RES, los aspectos formales guardan una cierta independencia respecto a los aspectos cognitivos, es decir que los aportes comparativos de contenido de ambos miembros no son siempre coincidentes con los aspectos formales. O sea que el mayor aporte cognitivo de un sujeto no necesariamente implica prevalencia en el aporte formal. Esto habla de una cierta independencia de la función grafémica y de escritura (función material) respecto de la función específicamente cognitiva. Esto no implica que la influencia cognitiva entre los sujetos desaparece, pero no necesariamente se traslada al plano de la operacionalidad formal o grafémica. Esto mismo se constató en un estudio anterior referido exclusivamente a MC (Roselli, 2017), donde el dibujo del MC, en sus aspectos formales, demostró ser bastante independiente de los aportes específicamente cognitivos.

\section{Discusión}

El propósito de la investigación fue esencialmente descriptivo: comparar las modalidades sociocognitivas diferenciales en la construcción colaborativa de MC y de RES. La hipótesis central que se asume es que dichos tipos de representación simbólica formatean, no sólo la apropiación cognitiva individual, sino también la interacción sociocognitiva en sí misma, es decir, la relación entre producción individual y colectiva, dando lugar a modalidades de construcción diferentes. 
El análisis consideró cuatro aspectos. En lo que hace a la cantidad de unidades cognitivas recuperadas del texto-fuente, RES demostró ser más exhaustivo. Su carácter exclusivamente lingüístico y secuencial, en comparación con la naturaleza estructural y plurirepresentacional de MC, puede explicar esta diferencia. En otras palabras, en MC habría un mayor filtrado de la información, y esto tanto en las producciones individuales, como en las colectivas.

El segundo aspecto analizado se refiere a la homogeneidad-heterogeneidad constatable entre las unidades cognitivas de las producciones individuales y colectiva de cada díada. Se encontró una tendencia de MC hacia una mayor homogeneidad y valores más altos de heterogeneidad en RES. En realidad, esta tendencia a la homogeneidad de MC no reside tanto en una diferencia de valores absolutos, sino en la proporción de elementos compartidos entre los dos sujetos de la díada respecto a los elementos no compartidos. En RES esta proporción es más asimétrica, predominando las unidades no-coincidentes intersubjetivamente (predominancia de la heterogeneidad). En parte esto se explica por la mayor presencia de unidades cognitivas recuperadas.

Pese a una menor heterogeneidad en MC, el tercer análisis realizado, referido a la simetría-asimetría de estos elementos no-coincidentes, puso en evidencia una asimetría intradíada más pronunciada en el caso de MC. En cambio, en RES, la proporción de unidades cognitivas nocoincidentes aportadas por ambos sujetos es más equitativa, o sea que hay una menor distancia entre los elementos que aporta el sujeto A y su par B. Como conclusión se podría decir que MC registra una notable cantidad de elementos coincidentes aportados por los dos sujetos (el núcleo duro de la información), pero una marcada asimetría interpersonal en el aporte de elementos nocoincidentes (los contenidos secundarios). En RES esta distribución de los elementos aportados por ambos sujetos es más armónica, o sea que la contribución individual a la construcción colectiva es más pareja, tanto en los contenidos centrales (en los que hay coincidencia), como en los contenidos más periféricos (en los que hay menos coincidencia).

Pero, quizás, la conclusión más relevante del segundo y tercer análisis sea que hay una diferencia marcada entre MC y RES al interior de varias díadas, tanto en la dimensión homogeneidad-heterogeneidad, como en la de la simetría-asimetría de la heterogeneidad. En efecto, el comportamiento de varias díadas es contradictorio en ambas dimensiones, según se trate de MC o RES. Por ejemplo, el sujeto A de una determinada díada es el que aporta más unidades cognitivas en MC, pero el sujeto B es el que prevalece en RES. Esto es aún más significativo si se tiene en cuenta que se trata de muestras relacionadas, o sea de comparaciones entre díadas integradas por los mismos sujetos. Las mismas díadas generan patrones diferenciales de interacción sociocognitiva, según se trate de MC o de RES.

Todo lo expuesto habla de un formateo interaccional diferenciado entre los dos sistemas externos de representación considerados. Cada uno de estos sistemas (MC y RES) no sólo produciría un formateo cognitivo individual (que no estuvo entre los objetivos centrales de este estudio analizar), sino también un formateo sociocognitivo o intersubjetivo, dando lugar a distintas modalidades de relación entre producciones individuales y colectivas.

En cuanto al análisis de los aspectos formales de las producciones, se encontró una marcada independencia de la función grafémica y de escritura (producción material del MC y RES colectivos) respecto a la función específicamente cognitiva o de contenido. En varios casos no es el sujeto que registra mayores aportes cognitivos el que está a cargo de la escritura en RES o el que aporta los mayores elementos formales en MC.

En síntesis, los MC producen una recuperación más selectiva y abreviada de la información del texto-fuente que los RES, tanto en las producciones individuales como colectivas; b) en lo que hace al aporte cognitivo de los miembros de la díada a la producción colectiva, los MC registran una mayor proporción de coincidencias cognitivas (homogeneidad) que de nocoincidencias (heterogeneidad); c) en dicho aporte de lo individual a lo colectivo los MC evidencian mayor asimetría en lo que hace a las contribuciones cognitivas, o sea que el aporte de 
los RES, aun siendo más heterogéneo, es más simétrico o equitativo; d) en cuanto a los aspectos formales, éstos son bastante independientes de los aspectos de contenido: no siempre el miembro que hace más aportes cognitivos es el que está a cargo de la operación de escritura o de confección grafémica.

Respecto a la cuestión de la simetría-asimetría de los aportes colaborativos los resultados son coincidentes con los de otras investigaciones (Buchs y Butera, 2009; Castellaro y Roselli, 2018), en el sentido que es una dimensión central a la hora de analizar la producción colectiva. La formación espontánea de las díadas en base a la afinidad socioafectiva sirvió para atenuar las posibles diferencias de competencia cognitiva inicial entre los miembros, tal como lo demostraron Castellaro y Roselli (2019) en una investigación sobre la comprensión colaborativa de tablas de frecuencias.

Con todo, se deben señalar varias limitaciones de la investigación. En primer lugar, es claro que la cantidad de díadas de las dos submuestras restringe las posibilidades de generalización de las conclusiones; sin duda se hace necesaria una replicación del estudio que permita ampliar el fundamento empírico y la significatividad estadística de las mismas. Por ello corresponde darle a este estudio un carácter preliminar o introductorio y un objetivo básicamente descriptivo dirigido a detectar tendencias. En segundo lugar, hubo un sesgo en cuanto de la distinta extensión (en términos de unidades cognitivas) de los dos textos-fuente utilizados que dificultó la comparabilidad entre mapas y resúmenes de las cuatro condiciones experimentales. Ello obligó a recurrir a coeficientes de relativización que permitieran equiparar valores absolutos. Sin duda, no es tarea fácil preparar textos epistémicos cognitivamente equivalentes, sobre todo si la investigación debe montarse en situaciones educativas y desarrollos curriculares reales.

\section{Referencias}

Aguilar Tamayo, M. F. (2006). El mapa conceptual: una herramienta para aprender y enseñar. Plasticidad y Restauración Neurológica, 5(1), 7-17.

Akkerman, S., Van den Bossche, P., Admiraal, W., Gijselaers, W., Segers, M., Simons, R., \& Kirschner, P. (2007). Reconsidering group cognition: From conceptual confusion to a boundary area between cognitive and socio-cultural perspectives? Educational Research Review, 2(1), 39-63.

Brizuela, B. M., \& Cayton, G. A. (2010). Anotar números desde pre-escolar hasta segundo grado: el impacto del uso de dos sistemas de representación en la presentación. Cultura y Educación, 22(2), 149-167. DOI: 10.1174/113564010791304500.

Buchs \& Butera (2009). Is a partner's competence threatening during dyadic cooperative work? It depends on resource interdependence. European Journal of Psychology of Education, 24(2), 145-154. DOI: 10.1007/BF03173007

Castellaro, M., \& Roselli, N. (2018). Resolución colaborativa de problemas lógicos en condiciones de simetría y asimetría cognitiva. Revista Propósitos y Representaciones, 6(1), 135-166.

Castellaro, M., \& Roselli, N. (2019). Simetría-asimetría cognitiva y afinidad socioafectiva en la comprensión colaborativa de tablas de frecuencias. Liberabit, 25(2), 213-231. DOI: https://doi.org/10.24265/liberabit.2019.v25n2.06

Kozulin, A. (2000). Instrumentos psicológicos: La educación desde una perspectiva sociocultural. Barcelona: Paidós.

Lorenzo, M. G., \& Pozo, J. I. (2010). La representación gráfica de la estructura espacial de las moléculas: eligiendo entre múltiples sistemas de notación. Cultura y Educación, 22(2), 231-246.

Lowe, R. K. (1993). Succesful instructional diagrams. Londres: Kogan Page.

Martí, E., \& Pozo, J. I. (2000). Más allá de las representaciones mentales: La adquisición de los sistemas externos de representación. Infancia y Aprendizaje, 90, 11-30.

Martí, E. (2000). Los mecanismos de internalización y externalización del conocimiento en las teorías de Piaget y Vigotsky. En: A. Tryphon y J. Voneche (comps), Piaget-Vigotsky: La génesis Social del Pensamiento. Buenos Aires: Paidós, Cap. 4, 81-113. 
Martí, E. (2003). Representar el mundo externamente. El desarrollo infantil de los sistemas externos de representación. Madrid: Antonio Machado.

Martí, E. (2017). Body, culture and cognition: avoiding reductionist temptations. Estudios de Psicología. DOI: 10.1080/02109395.2016.1268392

Mejía-Arauz, R., Rogoff, B., Dayton, A., \& Henne-Ochoa, R. (2018). Collaboration or negotiation: two ways of interacting suggest how shared thinking develops. Current Opinion in Psychology, 23, 117-123.

Notorio, A. (1992). El Mapa Conceptual como Técnica Cognitiva y su Proceso de Elaboración. Madrid: Narcea.

Pozo, J. I. (2017). Learning beyond the body: from embodied representations to explicitation mediated by external representations. Infancia y Aprendizaje / Journal for the Study of Education and Development, 40(2), 219-276.

DOI: https://doi.org/10.1080/02103702.2017.1306942

Puy Pérez-Echeverría, M., Martí, E., \& Pozo, J.I. (2010). Los sistemas externos de representación como herramientas de la mente. Cultura y Educación, 22(2), 133-147.

Puy Pérez-Echeverría, M., Postigo, Y., \& Marín, C. (2010). Las habilidades gráficas de los estudiantes universitarios: ¿Cómo comprenden las gráficas los estudiantes de psicología? Cultura y Educación, 22(2), 215-229.

Rivière, A. (1985). La Psicología de Vygotsky. Madrid: Aprendizaje Visor.

Rodríguez, J., Martí, E., \& Salsa, A. (2018). Symbolic representations and cardinal knowledge in 3- and 4-year-old children. Cognitive Development, 48(4), 235-243. DOI: https://doi.org/10.1016/j.cogdev.2018.09.004

Roselli, N. D. (1999). Identidad lógica y variabilidad representacional en tareas de resolución de problemas. Interdisciplinaria, Revista de Psicología y Ciencias Afines, 16(1), 75-97.

Roselli, N. D. (2017). Modalities to Collaborate in the Social Construction of Conceptual Maps: A Comparison between Individual and Collective Productions. American Journal of Educational Research, 5(10), 1058-1064.

Salsa, A., \& Gariboldi, M. B. (2017). Experiencia con símbolos y comprensión de dibujos en niños pequeños de distintos contextos socioeconómicos. Avances en Psicología Latinoamericana, 36(1), 29-43.

DOI: https://doi.org/10.12804/revistas.urosario.edu.co/apl/a.4332

Wertsch, J. (1988). Vygotsky y la Formación Social de la Mente. Barcelona: Paidos.

Wertsch, J. (1991). Voces de la Mente. Madrid: AprendizajeVisor. 


\section{ANEXO}

\section{$\underline{\text { Texto A }}$}

(los corchetes identifican las unidades cognitivas)

\section{La prevalencia de los rasgos mágicos y de las operaciones concretas en el pensamiento adulto}

[Según la concepción de Piaget, el desarrollo del concepto de causalidad está estrechamente vinculado al desarrollo de las estructuras lógico-formales.] [También suele pensarse que el hecho de alcanzar un nivel de pensamiento de carácter formal presupone la desaparición de las operaciones concretas.] [Sin embargo, existen estudios que demuestran que estas condiciones no siempre son válidas, lo cual invita a cuestionarnos al respecto.]

[Los autores Lesser y Painser (1985) han estudiado la continuidad del pensamiento mágico en los adultos de edades comprendidas entre los 25 y los 60 años.] [Evaluaron el nivel de pensamiento lógico formal alcanzado y sus nociones con respecto al concepto de causalidad.] [El estudio se realizó en dos grupos: uno pertenecía a una comunidad espiritual mientras que el otro consistía en adultos que no pertenecían a una comunidad espiritual.] [Los resultados demuestran que, si bien ambos grupos obtuvieron resultados similares en cuanto a las operaciones lógicoformales, se observaron diferencias en relación a los conceptos de causalidad.] [Así, ambos grupos difirieron en su concepción de cómo se explican los asuntos humanos;] [el grupo atípico (el de la comunidad espiritual) demostró tener orientación más proclive a una explicación mágica y una creencia más fuerte de lo sobrenatural, creyendo que en el universo cada suceso tiene su significado oculto.]

[De este modo, con este estudio se demuestra que existe cierta autonomía del concepto de causalidad con respecto al desarrollo de las estructuras lógico-formales.] [Es decir, que los conceptos mágicos sobre la causalidad se pueden desarrollar en la madurez y pueden convivir con estructuras formales de pensamiento.] [Rogoff (1984) también apoya esta idea, ya que cree que si se tiene en cuenta el contexto del sujeto y el sentido que el mismo le da a los sucesos, puede ocurrir que conductas consideradas como "ilógica" a los ojos del investigador convivan con las estructuras formales del adulto.]

[A su vez, el hecho de que se alcancen conductas cognitivas de carácter formal no quiere decir que desaparezcan las operaciones concretas.] [En un estudio realizado por Page (1985) se vio que como adultos, bajo el efecto de la hipnosis, mostraban un nivel de razonamiento moral inferior bajo condiciones de regresión con respecto a otros adultos de un grupo control;] [el nivel de razonamiento que alcanzaron era muy parecido a los niveles de razonamiento de los niños de edades de entre 9 y 15 años.] [De este modo, se demuestra que las operaciones concretas no desaparecen, sino que se mantienen a lo largo de la vida;] [el pensamiento lógico-formal se construye sobre las mismas y es esperable que ambos queden integrados.] 


\section{Texto B}

(los corchetes identifican las unidades cognitivas)

\section{$\underline{\text { Los prejuicios etnocéntrico y sexista }}$}

Hay otros problemas añadidos a los que acabamos de comentar. [Tal y como se definió en determinado momento el pensamiento adulto, encorsetándolo en el reducido marco del pensamiento lógico-formal, se perpetran dos injusticias notables: la primera de carácter etnocéntrico;] [la segunda es de carácter sexista.]

[El pensamiento lógico-formal, notable adquisición de la especie humana, es una creación occidental.] [La injusticia proviene de pensar que es la forma superior de pensar;] [además que tiene carácter universal.] [Allí donde no se observa es porque tal cultura no ha alcanzado su máximo nivel de desarrollo.] [Es decir, que otras formas socioculturales de pensar no son formas distintas, sino previas al pensamiento lógico-formal.]

[Piaget y García (1982) llaman la atención sobre, el hecho de que en el siglo V A.C. los chirlos habían formulado, de alguna forma, el principio de inercia;] [en cambio, los griegos hubieran considerado esta idea absurda.] [Dos concepciones del mundo diferentes conducen a explicaciones físicas distintas -no superiores o inferiores-, necesariamente.] [Las diferencias culturales producen marcos epistemológicos distintos.]

[Linn y Siegel (1984) piensan que el cambio que se produce en el pensamiento adulto se debe a que éste desplaza su centro de atención sobre el contexto -<< el marco epistemológico >.] [Con esto se abandona la estrategia de pura adquisición de conocimiento.] [Y comprende, además, cómo el contexto influye en el modo en que incorpora la nueva información.]

[El otro prejuicio al que me refería es el sexista.] [Aparte de ser el pensamiento lógicoformal una creación occidental, también es, en buena medida, desde el punto de vista cultural, una construcción masculina.] [Pero hay otras formas de pensar, igualmente complejas y potentes: en concreto, las creadas desde la óptica femenina.] [Las mismas son consideradas previas o anteriores al pensamiento lógico-formal y no son tenidas en cuenta.]

[Gilligan (1985) fue una destacada discípula de Kohlberg.] [Postula que no hay un único desarrollo moral al que se afilian del mismo modo tanto hombres como mujeres.] [Ella cree que el desarrollo moral de los varones progresa ininterrumpidamente hacia estadios de máxima complejidad en el marco de la ética de la justicia y el derecho.] [El desarrollo moral de las mujeres igualmente progresa ininterrumpidamente hacia operaciones de complejidad semejantes.] [Pero, en cambio, lo hacen en el marco de una ética de las responsabilidades, de la atención y del cuidado.]

[Consideraciones como las que se hacen aquí están conduciendo a muchos psicólogos del desarrollo a cambiar los presupuestos sobre los que se asentaban sus investigaciones.] [Parece que ya no es posible seguir afirmando que la lógica formal es la única que impregna el comportamiento intelectual de los adultos.] [Cada vez emergen con más nitidez desde el fondo de los resultados experimentales otras <<lógicas >> distintas -ni superiores, ni inferiores-.] [Sin el reconocimiento de esta variabilidad igualitaria el comportamiento adulto solo cabría interpretarlo como proceso degenerativo.] [Comentaremos, a continuación, solo dos: el pensamiento dialectico y el pensamiento cuántico-relativista.] 